\title{
A Conditional Simulation Method for Predicting Wind Pressure Fields of Large-Span Spatial Structures
}

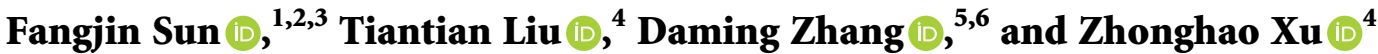 \\ ${ }^{1}$ Guangxi Key Laboratory of New Energy and Building Energy Saving, Guilin 541004, China \\ ${ }^{2}$ Guangxi Key Laboratory of Geomechanics and Geotechnical Engineering, Guilin 541004, China \\ ${ }^{3}$ College of Civil Engineering and Architecture, Guilin University of Technology, Guilin 541004, China \\ ${ }^{4}$ College of Civil Engineering, Liaoning Technical University, Fuxin 123000, China \\ ${ }^{5}$ Guangxi Key Laboratory of Embedded Technology and Intelligence, Guilin 541006, China \\ ${ }^{6}$ College of Information Science and Engineering, Guilin University of Technology, Guilin 541006, China
}

Correspondence should be addressed to Daming Zhang; e_horse@163.com

Received 30 August 2020; Revised 8 June 2021; Accepted 19 June 2021; Published 7 July 2021

Academic Editor: Sara Muggiasca

Copyright $\odot 2021$ Fangjin Sun et al. This is an open access article distributed under the Creative Commons Attribution License, which permits unrestricted use, distribution, and reproduction in any medium, provided the original work is properly cited.

Wind load is among the control loads for large-span spatial structures. Wind tunnel test is one of the commonly used methods for measuring wind pressure fields of different kinds of structures. However, due to the limited wind pressure data obtained from wind tunnel testing, it is quite meaningful to employ the limited measured data to predict the unknown wind pressure at target points. Considering the complexity of wind pressure fields of large-span spatial structures, a simplified nonparametric method based on conditional simulation is proposed to predict the unknown pressures using the existing data. The Karhunen-Loève (KL for short) expansion is employed to represent wind pressure random variants as eigenfunctions of the covariance operator. To reduce the variant dimensionality, the nearest neighboring estimator is given for the transition distribution of the KL expansion. The targeted wind pressure fields are obtained by expanding the Fourier basis of the eigenfunction and estimating its expansion coefficients. The proposed method is applied to estimate wind pressures on a gable roof building. The relevant parameters of the wind pressure field are obtained, and the results compare well with those from wind tunnel testing, with higher efficiency. The proposed method effectively reduces the dimensionality of the predicted wind pressures, with reduced errors, higher accuracy, and increased efficiency.

\section{Introduction}

Wind load is one of the control loads for large-span spatial structures. The current structural design specifications [1] are not specified for the calculation of the wind pressure of this type of structure. Usually, the most reliable method for measuring the wind pressure of large-span roofs is to conduct wind tunnel testing. But the surface of large-span roof structures generally needs to be arranged 800 to 1000 measurement points, sometimes even more. However most research institutions rarely have electronic scanner equipment that can measure such a large number of measurement points [2], the measured wind pressure coefficient is often limited. Then, under the conditions of limited and known wind pressure field data, the wind pressure field without test points (locations) is accurately and efficiently simulated; that is, conditional simulation, for accurately predicting the wind-induced response of large-span roofs, has important value and significance for its wind-resistant design.

At present, there are mainly two kinds of conditional simulation methods [2]: the Kriging method and the conditional probability density function method. The Kriging method is mainly through the random variable to be estimated expressing as a linear combination of several known reference variables and the corresponding correlation function. The conditional probability density function method was first proposed by Kamenda and Morikawa, applied to earthquake engineering [3]. This method mainly uses the joint probability distribution function between variables to estimate unknown variables. Because the wind 
pressure field of a large-span roof is more complicated, if the above two methods are directly adopted, it may cause a huge amount of calculation and the solution is easily divergent. In recent years, a technique called "function principal component analysis" [4] can be used to limit the value of random functions in separable Hilbert spaces and perform orthogonal basis functions (which is the eigenfunction containing the covariance operators); the extended method reduces the function dimension, to reduce the amount of calculation. The so-called principal components are those corresponding to the selected first few basis functions. Much of the current research uses the "function principal component analysis" technique to estimate the principal components [5-7], but a few studies use the estimated principal components for prediction. If it is used for prediction, due to the dimension of the random function decreases, it will effectively reduce the amount of calculation and improve the calculation accuracy and efficiency. Domestic scholars mainly use the artificial neural network (ANN) $[8,9]$ and proper orthogonal decomposition (POD) method $[10,11]$ to predict the wind pressure field of the structure in terms of wind pressure field simulation. Samaniego and Anitescu explored Deep Neural Networks (DNNs) [12] to solve boundary value problems. The authors considered two approaches: a collocation method and the Deep Energy Method. An important implementation aspect that emerges is that the loss function for the DNN can be naturally devised from the energy of the body. Several very relevant examples from computational mechanics have been solved using DNNs to build the approximation space, which shows that it is possible to tackle the solution of very relevant BVPs using concepts and tools coming from deep learning.

Considering the complexity of the random variables of the wind pressure field of large-span spatial structures, this paper proposes a conditional simulation method of wind pressure using the nearest neighbor estimation of the function transition distribution, which is also a nonparametric conditional simulation method. This method first uses Karhunen-Loève (abbreviated as KL) to expand the wind pressure random variable as the eigenfunction of the covariance operator, and then the nearest neighbor estimate of the KL extended transition distribution is given. Then, the expansion coefficient is estimated by extending the Fourier basis of the eigenfunction to realize the estimation of the eigenfunction and obtain the target wind pressure value. Finally, this method is applied to the estimation of the wind pressure field of the large-span structures.

\section{Conditional Simulation Method}

The assumption of Gaussian wind loading is not however always correct as pointed out by previous researchers. In those cases, the conditional simulation of a non-Gaussian wind velocity field is necessary [13]. Combine the conditional simulation technique of Gaussian random fields by Behrouz [14] and the iterative procedure for the unconditional simulation of non-Gaussian random fields [15] to conditionally simulate the time-independent non-Gaussian random fields. Gurley and Kareem [16] developed a procedure for the conditional simulation of multivariate non-Gaussian velocity/pressure fields. For mapping a Gaussian process to a non-Gaussian process and vice versa, they used a modified Hermite transformation using the Hermite polynomial function. The simulated process generated by this method compares well with the target one up to the fourth-order of statistics.

Based on the known $(i-1)$ wind pressure test records $P_{(i-1)}$, the conditional simulation to predict the wind pressure $P_{i}$ at the ith point can be written as [17]

$$
P_{i}(v)=\sum_{n=1}^{i-1} \int_{v} \Lambda(v, u) P_{n}(v)+K_{i}(v),
$$

where $u, v \in \Phi, \Phi$ is a compact subset of $\mathfrak{R}^{k}(k \geq 1) ; K_{i}$ is a random variable whose value is on a separable Hilbert space, and $\Lambda_{i}$ is a sequence of the linear random operator. It is assumed that the wind pressure at each measurement point of $i$ is independent and uncorrelated, and then

$$
\Lambda_{i}(v, u)=\sum_{s \in N} W_{i, s} \delta_{s}(v) \delta_{s}(u)
$$

where $\left\{W_{i, s} ; s \in N\right\}$ is an independent random variable, $\delta_{s}$ is an orthogonal real-valued eigenfunction of a set of integral operators, its kernel function is written as

$$
C_{0}(u, v)=\sum_{s \in N} \gamma_{S} \delta_{S}(u) \delta_{s}(v), \quad \gamma_{s} \geq \gamma_{s+1} \ldots
$$

where $\gamma_{s}$ is the real-valued order eigenvalue.

Then, in the $L_{2}$ separable domain of Hilbert space, the wind pressure $P_{i}$ can be expanded by Karhunen-Loève (KL) as

$$
P_{i}(v)=\sum_{s \in N} Q_{i, s} \delta_{s}(v)
$$

where $Q_{i, s}$ is a series of unrelated random variables, and there are [17]

$$
Q_{i, s}=\int_{v} P_{i}(v) \delta_{s}(v) \mathrm{d} v=\left\{P_{i}, \delta_{s}\right\}
$$

\section{Conditional Simulation for Wind Pressure Fields of Large-Span Roofs Based on Consistent Estimation}

3.1. Transition Distribution and Its Nearest Neighboring Estimation. To improve the efficiency and accuracy of wind pressure field simulation, the method of reducing the dimension of wind pressure field variables is employed, which is to map high-dimensional data to low-dimensional space and maintain the properties of the original data. The optimization of these high-dimensional data usually falls into the category of eigenvalue estimation. The KL expansion of random wind pressure field variable is represented by adopting the eigenfunction of covariance operator; then, a simple nonparametric model is proposed. By considering one-dimensional nearest neighboring estimation [18] of arbitrary coefficient transition distribution in KL expansion, 
wind pressure field variable dimension is reduced. Therefore, the transition distribution of the wind pressure field $P_{i}(v)$ and its nearest neighboring distribution have to be found firstly, and then eigenfunction will be estimated. Reducing the dimension of the wind pressure field will be discussed from the two aspects, that is, the nearest neighboring estimation and eigenfunction estimation.

Estimation of the $\left(P_{i}(v)\right)_{i=1}^{n}$ transition distribution can be written as

$$
U_{n}(p(v) \mid x):=\int_{\Re}\left\{\sum_{s=1}^{S} \widehat{\delta}_{s}(v) q_{s} \leq p(v)\right\} \prod_{s=1}^{S} d U_{s n}\left(q_{s} \mid K_{s}\left(<x, \widehat{\delta}_{s}>, r_{s}\right)\right) a,
$$

where $\left[\widehat{\delta}_{s}(\cdot), s=1, \ldots, S\right]$ is the orthogonal estimator of $\left[\delta_{s}(\cdot), s=1, \ldots, S\right], P_{i}(v)$ is the wind pressure field, $q_{s}$ is the element of the random variable, and $r_{s}$ is a family radius established around $x_{m}=\left\langle Y_{n}, \widehat{\delta}_{s}\right\rangle$ for each as $s=1, \ldots, S$. The equation shows that when estimating the wind pressure field, the number of $s$ one-dimensional estimated value is needed instead of a multidimensional variable, to reduce the calculation time and improve the efficiency. Next is how to construct the one-dimensional estimation. The one-dimensional nearest neighboring estimator of the transition distribution is used to reduce dimensions since the nearest neighboring estimator can maintain the characteristics of the original data well and easy to get.

Since general combinations of linear functions are adopted in equation (6), for any Lipschitz function g: $\mathfrak{R} \longrightarrow \mathfrak{R}$ can be written as

$$
\int g(p(v)) U_{n}(p(v) \mid x)=\sum_{i=2}^{n}\left[\prod_{s=1}^{s} \frac{\left\{Q_{i-1, s n} \in K_{s}\left\langle x_{s, n}, r_{s}\right\rangle\right\}}{\sum_{i=2}^{n}\left\{Q_{i-1, s n} \in K_{s}\left\langle x_{s, n}, r_{s}\right\rangle\right\}}\right] g\left(\sum_{s=1}^{s} Q_{i, s n} \widehat{\delta}_{s}(v)\right),
$$

where $x_{s, n}:=\left\langle x, \widehat{\delta}_{s}\right\rangle, r_{s}$ is a family radius established around $x_{m}=\left\langle Y_{n}, \widehat{\delta}_{s}\right\rangle$ for each as $s=1, \ldots, S$.

The nearest neighboring estimator is an anticlustering method to reduce the variable dimensions. The basic idea of seeking the nearest neighboring estimator is to calculate the distance between the target center and the neighboring point and control it within the expected value; then, the nearest neighboring estimator of $U_{s}\left(q_{s} \mid x_{s}\right)$ can be defined as

$$
U_{s n}\left(q_{s} \mid K_{s}\left(\left\langle x, \widehat{\delta}_{s}\right\rangle, r\right)_{s}\right):=\frac{\sum_{i=2}^{n}\left\{\widehat{\delta}_{s}, P_{i-1} \in K\left(\left\langle x, \widehat{\delta}_{x}\right\rangle, r_{s}\right),\left\langle\widehat{\delta}_{x}, P_{i}\right\rangle \leq q_{s}\right\}}{\sum_{i=2}^{n}\left\{\left\langle\widehat{\delta}_{s}, Y_{i-1}\right\rangle \in K\left(\left\langle x, \widehat{\delta}_{s}\right\rangle, r_{s}\right)\right\}},
$$

where $q_{s}$ is the element of the random variable and $Q_{i, s}$ is the wind pressure field at the known measuring point.

Next, estimating the orthogonal eigenfunction of the covariance operator $\left[\delta_{s}(\cdot), s=1, \ldots, S\right]$ will be discussed in Section 3.2.

3.2. Eigenfunction Estimation. It can be seen from equation (4) that the key to simulate the wind pressure field conditions is to seek the integral operator eigenvalue $\delta_{s}(v)$ of core function $C_{0}(u, v)$ in the entire $\Phi$ domain. Arbitrary variables are needed.

Assuming $\left[\widehat{\delta}_{s}(\cdot), s=1, \ldots, S\right]$ is the orthogonal estimators of $\left[\delta_{s}(\cdot), s=1, \ldots, S\right]$, then

$$
\left|\widehat{\delta}_{s}-\delta_{s}\right|=O_{p}\left(\prod_{s}^{-1} n^{-\tau}\right)
$$

where $\tau>0\left[\prod_{s}: s=1, \ldots, S\right]$ depends on the spacing between eigenvalues.
Based on the above conditions, the wind pressure simulation method proposed mainly involves Fourier basis expansion of the eigenfunctions and the expansion coefficient estimation, that is, the estimation of the eigenfunction. Generally, based on the interval $\Phi=[0,1]$, the covariance function based on the binary Fourier basis can be written as

$$
C 0(u, v)=\sum_{r, l \in Z} c(r, l) \exp \{i(r u+l v)\}
$$

where $c(r, l)$ is the $(r, l)$-th Fourier coefficient of the kernel function $C_{0}(u, v)$. The estimation of the Fourier basis expansion coefficients of the eigenfunctions can be obtained by truncating between $-S$ and $S$ through the above Fourier expansion. Based on the Mercer theory, the Fourier basis expansion coefficient estimation can be written as

$$
\delta_{s, n}^{S}(v)=\sum_{r=-S}^{S} a_{s, n}(r) \exp (\mathrm{irv})
$$


where $a_{s, n}(r)$ is the $r$-th input of the $s$-th eigenvector $C_{n}^{s}$ of the Fourier coefficient $c_{n}^{s}(r, p)$, and $c_{n}^{s}(r, p)$ is obtained by the truncated two-dimensional Fourier expansion of the estimated covariance function, an $S \times S$ order matrix, expressed as

$$
c_{n}^{S}(u, v)=\sum_{R=-S}^{S} \sum_{P=-S}^{S} c_{n}^{S}(r, p) \exp \{i(r u+p v)\} .
$$

3.3. Conditional Simulation Procedures for Wind Pressure Prediction of Large-Span Roofs. According to the above, conditional simulation procedures for wind pressure prediction of large-span roofs can be summarized as follows:

(1) According to equations (10) and (11), the first $s$ orthogonal eigenfunctions $\left\{\delta_{s}(\cdot), s=1, \ldots, S\right\}$ of the covariance operator are estimated and to make sure equation (9) is satisfied.

(2) According to wind pressure data measured in the experiment, the estimated eigenfunction $\left\{\left\langle P_{i}, \widehat{\delta}_{s}\right\rangle, i=1, \ldots, n ; s=1, \ldots, S\right\}$ is obtained.

(3) Equations (6) and (7) are adopted to calculate the transition distribution of each known wind pressure measuring point, and then equation (8) is used for the nearest neighboring estimator. That is, $\left\{\delta_{s}(\cdot), s=\right.$ $1, \ldots, S\}$ is estimated firstly, and the known wind pressure of measuring points is transformed onto two-dimensional space $\left\{\left(Q_{i, s n}\right)_{i=1}^{n}:\right\}$ through $\left\{\left\langle P_{i}, \delta_{s}\right\rangle, i=1, \ldots, n ; s=1, \ldots, S\right\}$.

(4) The estimated value of the random variable $\left.\left\{\left(Q_{i, S n}\right)\right)_{i \in\{1, \ldots, n\}} ; s=1, \ldots, S\right\}$ is obtained by equation (12), using the eigenfunction $\left\{\widehat{\delta}_{s}: s=1, \ldots, S\right\}$, where $Q_{i, s n}:=Q_{i, s, n}=\left\langle P_{i}, \widehat{\delta}_{s}\right\rangle$, and finally the wind pressure $P_{(n+1)}$ is obtained by equations (4) and (5).

\section{Example Analysis}

In this paper, the wind pressure field of a double-sloped large-span roof structure is simulated by the above method. The roof sketch and the arrangement of some measuring points are shown in Figure 1 [19]. A total of 84 pressure measuring points are arranged on the roof in the wind tunnel test. In the experiment, the roof is divided into four areas noted 1 to 4 (as shown in Figure 2). According to the division of the roof areas in the experiment [19], the wind pressure coefficients of the measuring points 74-80 (except point 79) in areas 1 and 2 are used as the known data of the conditional simulation in the experiment. The wind pressure-related parameters of the measuring points 79,81 , to 84 in areas 3 and 4 are predicted through the proposed method. The results are compared with the wind tunnel experimental data to validate the correctness of the proposed method.

When using the method introduced in this article to calculate, mainly follow the steps in Section 3.3. In the specific implementation process, for the convenience of calculation, first, establish a set of finite grid points for each known wind pressure and sum all the grid points, that is, the integral of equation (7) is replaced by equation (13). That is, for each $s, s=1, \ldots, S$ establishes a circle with a radius $r_{s}=$ $c_{s} \alpha_{s} n^{-1 / 4}$ around $x_{s n}=\left\langle P_{n}, \widehat{\delta}_{s}\right\rangle$, and $c_{s}$ is an adjustment constant, where $c_{s}=1.8[14], S=7$. Then, equation (8) is used to establish the nearest neighbor estimation of the transfer distribution for each known wind pressure value, and the wind pressure value of the predicted point can be obtained by replacing $\left(Q_{n+1, s}\right)_{s=1}^{S}$ in the KL expansion. This method is also applicable to other examples of wind pressure prediction. The number of samples can be increased according to the required accuracy. However, the subsequent calculation results of this paper show that even under the condition of limited samples, the calculation accuracy can be guaranteed:

$$
\int p(v) d U_{n}(P(v \mid x))=\sum_{s=1}^{s} \bar{Q}_{s n} \widehat{\delta}_{s}(v) .
$$

Among them,

$$
\bar{Q}_{s n}=\sum_{i=2}^{n} Q_{i, s n} \frac{\left\{Q_{i-1, s n} \in K_{s}\left(x_{s, n}, r_{s}\right)\right\}}{\sum_{i=2}^{n}\left\{Q_{i-i, s n} \in K_{s}\left(x_{s, n}, r_{s}\right)\right\}} .
$$

Table 1 shows the average wind pressure coefficient of the predicted point at $0^{\circ}$ wind direction angle calculated by this method and compared with the experimental data [19].

Table 2 shows the root mean square value of the pulsating wind pressure coefficient at the predicted point at the $0^{\circ}$ wind direction angle calculated by the method in this paper and compares it with the experimental data [19].

As shown in Tables 1 and 2, the mean wind pressure coefficient and root mean square value of fluctuating wind pressure coefficient of the predicted taps simulated by this method are close to the experimental value, and the error is small, indicating the accuracy of this simulation method is good. It is should be noticed that despite the limited number of samples, the simulation accuracy is still satisfactory. It can be concluded that, if the samples increase, the simulation accuracy will be improved further. To compare the effects of the number of samples on the accuracy of the calculation, all the taps from 73 to 83 are used as the known data for the conditional simulation. The average wind pressure coefficients and the root mean square values of the fluctuating wind pressure coefficients from the conditional simulation of tap 84 are compared with the results when taps 74 to 80 (except for tap 79) are used as conditional simulation samples. It is found that the accuracy of the predicted values is increased by $3 \%$ and $5.3 \%$, respectively, while the calculation time increased by $11.9 \%$ and $9.6 \%$, respectively. Therefore, while increasing conditional simulation samples to improve the computation accuracy, it is also necessary to consider the computation time cost and strive to make a balance between the two.

To illustrate the superiority of the method in the work here, the mean absolute error (MAE) and the mean square error (SAE) of the predicted points at $90^{\circ}$ wind direction angle are calculated using the proposed method and compared with the calculation errors of two other methods (LA 


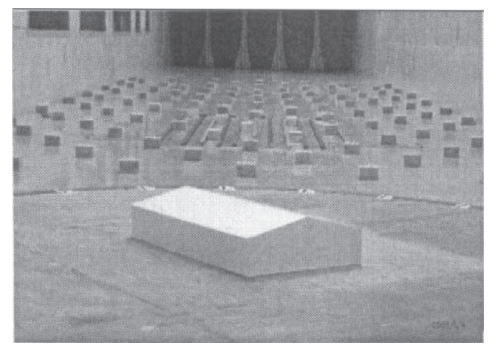

(a)

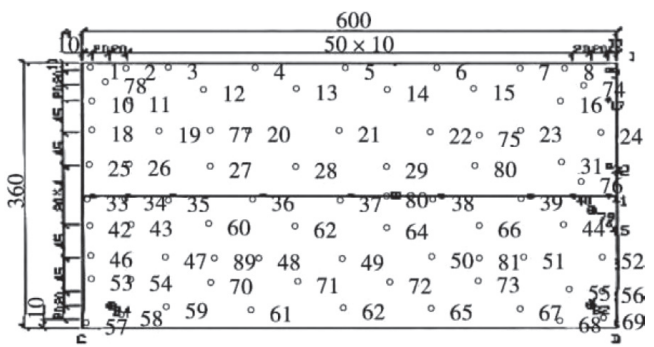

(b)

FIgURE 1: Wind tunnel experiment model and pressure tap layout for a gable roof [19].

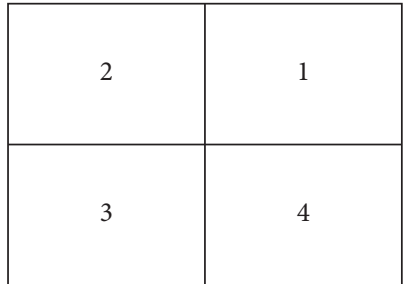

Figure 2: Roof divided zones.

TABle 1: Comparison of mean wind coefficients between conditional simulation and experiment at $0^{\circ}$ wind azimuth.

\begin{tabular}{lccc}
\hline Prediction points & Experiment value & Analog value & Error (\%) \\
\hline 79 & -0.4833 & -0.5018 & 3.83 \\
81 & -0.1224 & -0.1173 & 4.17 \\
82 & -0.5284 & -0.4987 & 5.62 \\
83 & -0.0190 & -0.0179 & 5.79 \\
84 & -0.0294 & -0.0278 & 5.44 \\
\hline
\end{tabular}

TABLE 2: Comparison of fluctuating wind coefficients RMS between conditional simulation and experiment at $0^{\circ}$ wind azimuth.

\begin{tabular}{lrcc}
\hline Prediction points & Experiment value & Analog value & Error (\%) \\
\hline 79 & -0.1545 & -0.1649 & 4.15 \\
81 & -0.0978 & -0.0952 & 2.65 \\
82 & -0.1726 & -0.1804 & 4.52 \\
83 & -0.0380 & -0.0401 & 5.52 \\
84 & -0.0330 & -0.0318 & 3.63 \\
\hline
\end{tabular}

and PF), commonly used in conditional simulation, the number of simulations in the three methods is $n=100$, and the results are shown in Table 3. Among them,

$$
\operatorname{MAE}=\frac{1}{N} \sum_{i=1}^{N}|\varepsilon(i)| / \text { SAE }=\frac{E\left\{[\varepsilon(i)]^{2}\right\}}{E\left\{\left[y(i)-E(\hat{y}(i))^{2}\right]\right\}},
$$

where $\varepsilon(i)$ is the output error, $\varepsilon(i)=y(i)-\hat{y}(i)$, $i=1, \ldots, N, y(i)$ is the experiment wind pressure coefficient, and $\hat{y}(i)$ is the conditional simulation results.

The LA method [20] refers to the linear autoregressive estimation method, and the PF method [21] refers to the prediction factor estimation method. The disadvantages of these two methods are that the simulation results are
TABLE 3: Comparison of errors by different conditional simulation methods at $90^{\circ}$ wind azimuth.

\begin{tabular}{|c|c|c|c|c|c|}
\hline \multirow[t]{2}{*}{ Prediction points } & \multirow[t]{2}{*}{ Adapting methods } & \multicolumn{2}{|c|}{$\begin{array}{l}\text { Average } \\
\text { wind } \\
\text { pressure } \\
\text { coefficient }\end{array}$} & \multicolumn{2}{|c|}{$\begin{array}{l}\text { Pulsating } \\
\text { wind } \\
\text { pressure } \\
\text { coefficient }\end{array}$} \\
\hline & & MAE & SAE & MAE & SAE \\
\hline \multirow{3}{*}{79} & LA & 2.652 & 0.286 & 2.739 & 0.336 \\
\hline & PF & 2.296 & 0.227 & 2.527 & 0.277 \\
\hline & Article method & 1.031 & 0.152 & 1.224 & 0.142 \\
\hline \multirow{3}{*}{81} & LA & 3.547 & 0.322 & 3.314 & 0.353 \\
\hline & $\mathrm{PF}$ & 2.234 & 0.269 & 2.531 & 0.291 \\
\hline & Article method & 1.227 & 0.126 & 1.471 & 0.126 \\
\hline \multirow{3}{*}{82} & LA & 2.459 & 0.332 & 3.753 & 0.416 \\
\hline & $\mathrm{PF}$ & 2.631 & 0.341 & 3.354 & 0.316 \\
\hline & Article method & 1.162 & 0.137 & 1.376 & 0.225 \\
\hline \multirow{3}{*}{83} & LA & 2.937 & 0.326 & 3.144 & 0.397 \\
\hline & $\mathrm{PF}$ & 2.358 & 0.402 & 2.528 & 0.326 \\
\hline & Article method & 1.265 & 0.113 & 1.176 & 0.206 \\
\hline \multirow{3}{*}{84} & LA & 2.941 & 0.428 & 2.756 & 0.376 \\
\hline & $\mathrm{PF}$ & 2.438 & 0.331 & 2.173 & 0.313 \\
\hline & Article method & 1.296 & 0.201 & 1.143 & 0.176 \\
\hline
\end{tabular}

accurate only when the model that generates the simulated data meets the linear assumption. In other words, the accuracy of the simulation results needs to be improved for the presence of nonlinear factors. Considering the nonlinear factors, the kernel function is introduced in the method in this paper. The kernel function is a key factor for implicitly processing nonlinear data in the feature space. The calculation in the feature space will be simple and efficient by using the kernel function.

It can be seen from Table 3 that the calculation error of this method is much lower than that of the two commonly used conditional simulation methods. Among them, the calculation accuracy of the average absolute error of this method is about $51 \%$ higher than that of the LA method, and about 36\% higher than that of the PF method. The calculation accuracy of the mean square error is improved by about $58 \%$ on average compared with the LA method, and about $47 \%$ on average compared with the PF method. It shows that the method in this paper is correct and reliable under different wind direction angles.

At the same time, time histories of the predicted target taps are shown with the conditional simulation through 


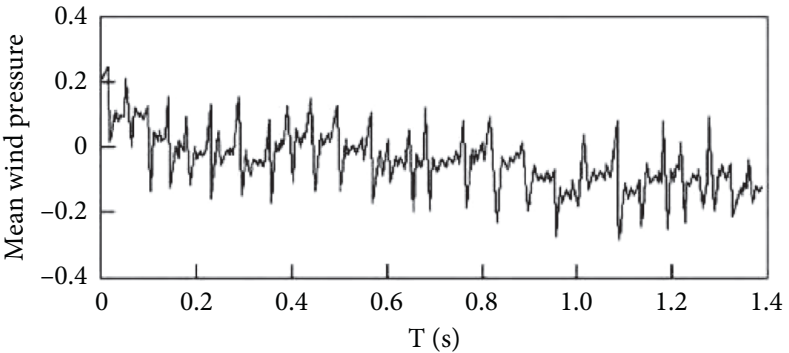

(a)

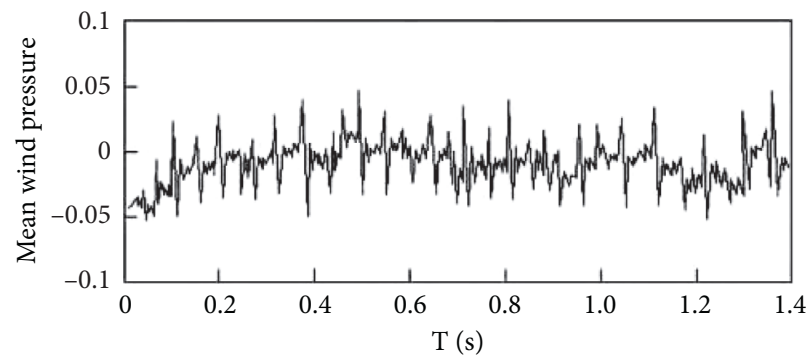

(c)

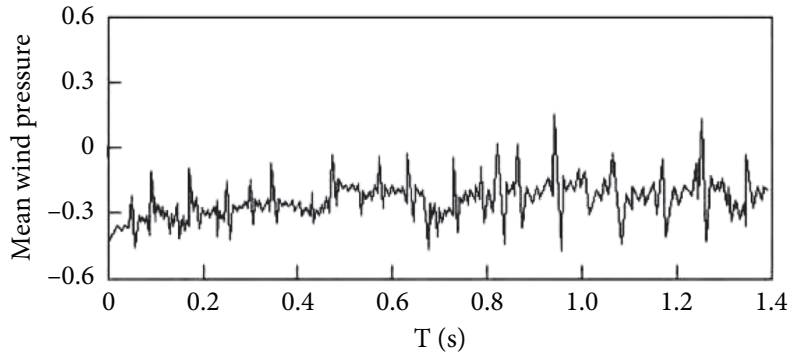

(b)

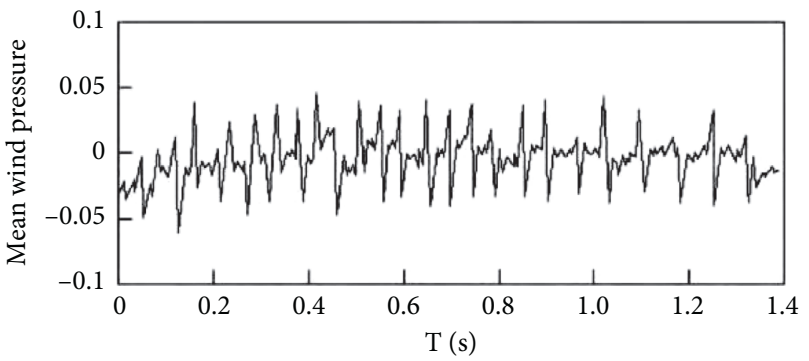

(d)

Figure 3: Time histories of wind pressure at predicted points. (a) 81 points. (b) 82 points. (c) 83 points. (d) 84 points.

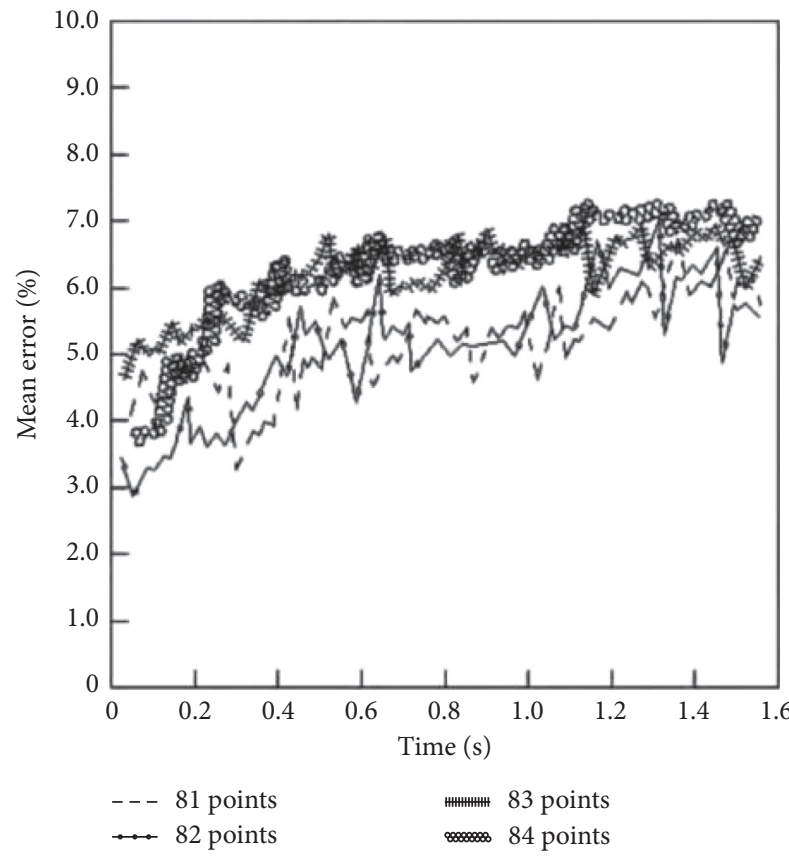

(a)

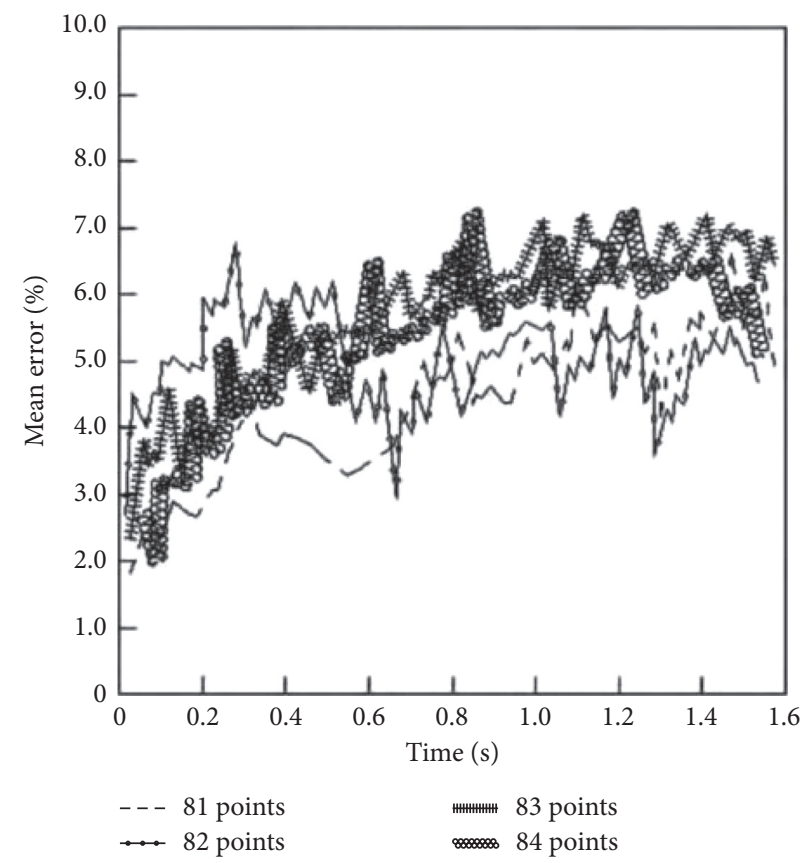

(b)

FIGURE 4: Variation of mean wind pressure coefficient errors. (a) Mean error change. (b) Root mean square error variation.

wind pressure coefficients of the known pressure taps, as shown in Figure 3.

To compare the predicted wind pressure with the experimental data and to validate the accuracy of the conditional simulation, the average error and root mean square error of the simulated average wind pressure coefficients are given, as shown in Figure 4.
It can be seen from Figure 4 that the mean error and root mean square error of the wind pressure coefficients simulated varied little with time, and the errors are basically below $8 \%$, which validates the accuracy of the simulation.

To illustrate the efficiency of the conditional simulation, the residuals of the two commonly used conditional simulation methods are compared, as shown in Figure 5. 


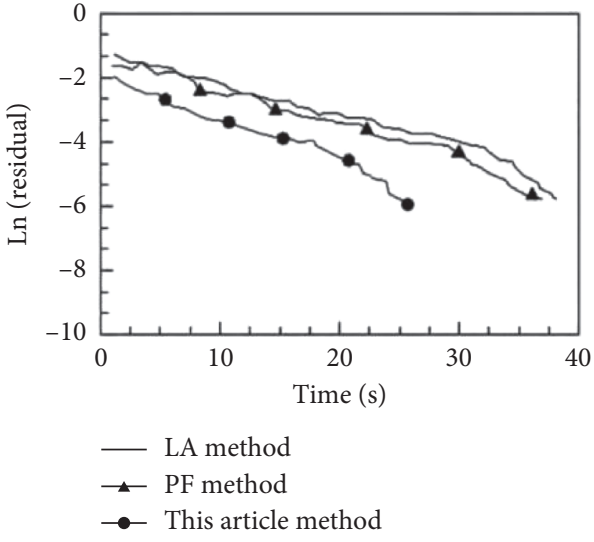

(a)

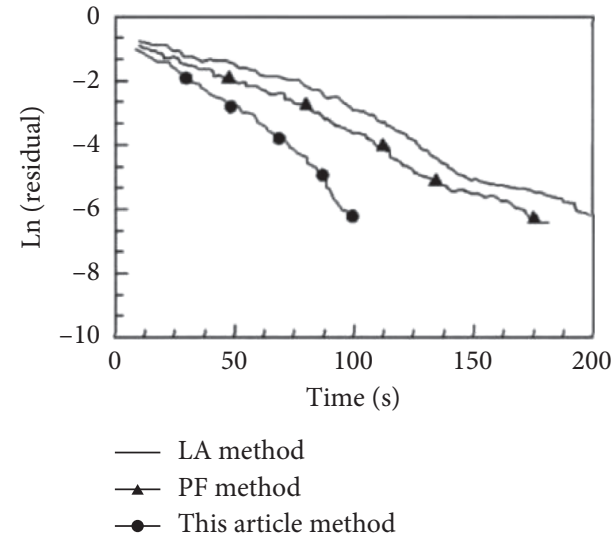

(b)

FIGURE 5: Comparison of residuals by different conditional simulation methods. (a) Calculate the residual variation with time. (b) Calculated residual varies with the number of simulations.

It can be seen from Figure 5 that, with the same convergence accuracy achieved, the time and the number of simulations required by the proposed method in this work are less than those of the other two conditional simulation methods, which indicates the efficiency of the proposed method. The reason why the residual decreases with the number of simulations is that as the number of simulations increases, the computation convergence accelerates. This is because the nearest neighbor estimation reduces the originally high-dimensional variables to one-dimensional variables, which improves the calculation efficiency; on the one hand, the use of the kernel function speeds up the calculation of the eigenfunction $\left\{\delta_{s}: s=1, \ldots, S\right\}$, since the use of the kernel function improves the two-dimensional space $\left\{\left(Q_{i, s n}\right)_{i=1}^{n}: s=1, \ldots, S\right\}$ "similarity" identification of the variables, which speeds up the calculation and convergence.

\section{Conclusions}

This paper proposes a nonparametric conditional simulation method of wind pressure using the nearest neighbor estimation of the function transfer distribution. The proposed method is applied to the prediction of wind pressure field on large-span roofs, and the main conclusions obtained are as follows:

(1) The average wind pressure coefficient and pulsating wind pressure coefficient of the large-span roof predicted by the method in this paper are in good agreement with the experimental values. The mean error and root mean square error of the wind pressure coefficient have little change with time and are basically below $8 \%$, which proves that the method proposed in this paper has high accuracy in simulating the wind pressure field.

(2) In this method, the error of the prediction result of the wind pressure field of a large-span roof is much smaller than that of the commonly used conditional simulation method applicable to linear models. The calculation accuracy of the average absolute error of this method is about $51 \%$ higher than that of the LA method and approximately $36 \%$ higher than the PF method; the calculation accuracy of the mean square error is about $58 \%$ higher than the LA method, and about $47 \%$ higher than the PF method; and the accuracy of the simulation method is not affected by the number of samples.

(3) The calculation accuracy and convergence speed of the method in this paper are higher than the general conditional simulation method. This is due to the fact that the use of nearest neighbor estimation reduces the variable dimension and the kernel function speeds up the eigenfunction computation.

\section{Data Availability}

The data used to support the findings of this study are available from the corresponding author upon request.

\section{Conflicts of Interest}

The authors declare that they have no conflicts of interest.

\section{Acknowledgments}

This study was supported by the National Natural Science Foundations of China (no. 51108345), Guangxi Key Laboratory of Geomechanics and Geotechnical Engineering (GUIKENENG19-Y-21-2), Guangxi Key Laboratory of New Energy and Building Energy Saving Foundation (Gui Keneng 19-J-21-14), Open Foundation of Guangxi Key Laboratory of Embedded Technology and Intelligence (no. 2019-02-08), Joint Cultivation Program of National Natural Science Foundations of Guangxi (2019GXNSFAA245037), Guangxi Youth Innovative Talents research project (Guike AD19245012), and Scientific and Technology Startup Foundation of Guilin University of Technology (GUTQGJJ2019042 and GUTQDJJ2019041). 


\section{References}

[1] Standards Press of China, GB 50009-2012 Code for the Design of Building Structure, Standards Press of China, Beijing, China, 2012, in Chinese.

[2] C. Zhou and N.-C. Xiao, "An active learning method combining kriging and probability density function for efficient reliability analysis," Proceedings of the Institution of $\mathrm{Me}$ chanical Engineers, Part O: Journal of Risk and Reliability, vol. 235, pp. 1-14, 2019.

[3] H. Kameda and H. Morikawa, "An interpolating stochastic process for simulation of conditional random fields," Probabilistic Engineering Mechanics, vol. 7, no. 4, pp. 242-254, 1992.

[4] J. Goldsmith, V. Zipunnikov, and J. Schrack, "Generalized multilevel function-on-scalar regression and principal component analysis," Biometrics, vol. 71, no. 2, pp. 344-353, 2015.

[5] Y. Li and T. Hsing, "Uniform convergence rates for nonparametric regression and principal component analysis in functional/longitudinal data," Annals of Statistics, vol. 38, no. 6, pp. 3321-3351, 2010.

[6] Y. Aït-Sahalia and D. Xiu, "Using principal component analysis to estimate a high dimensional factor model with high-frequency data," Journal of Econometrics, vol. 201, no. 2, pp. 384-399, 2017.

[7] L. SHōrmann and R. Horvàrth, "Reeder," Econometric Theory, vol. 29, no. 2, pp. 267-288, 2013.

[8] A. Afram, F. Janabi-Sharifi, A. S. Fung, and K. Raahemifar, "Artificial neural network (ANN) based model predictive control (MPC) and optimization of HVAC systems: a state of the art review and case study of a residential HVAC system," Energy and Buildings, vol. 141, pp. 96-113, 2017.

[9] S. U. Ning and P Shitao, "Research and application of neural network modeling of wind load on the main structure of large-span coal shed," Journal of Building Structures, vol. 7, pp. 34-41, 2019, in Chinese.

[10] M. S. Siddiqui, S. T. M. Latif, M. Saeed, M. Rahman, A. W. Badar, and S. M. Hasan, "Reduced order model of offshore wind turbine wake by proper orthogonal decomposition," International Journal of Heat and Fluid Flow, vol. 82, no. 82, Article ID 108554, 2020.

[11] A. Mohammadi and M. Raisee, "Stochastic field representation using bi-fidelity combination of proper orthogonal decomposition and Kriging," Computer Methods in Applied Mechanics and Engineering, vol. 357, Article ID 112589, 2019.

[12] E. Samaniego, C. Anitescu, S. Goswami et al., "An energy approach to the solution of partial differential equations in computational mechanics via machine learning: concepts, implementation and applications," Computer Methods in Applied Mechanics and Engineering, vol. 362, Article ID 112790, 2020.

[13] D. Karmakar, S. Ray-Chaudhuri, and M. Shinozuka, "Conditional simulation of non-Gaussian wind velocity profiles: application to buffeting response of Vincent Thomas suspension bridge," Probabilistic Engineering Mechanics, vol. 29, no. 29, pp. 167-175, 2012.

[14] B. Shafei and A. Alipour, "Application of large-scale nonGaussian stochastic fields for the study of corrosion-induced structural deterioration," Engineering Structures, vol. 88, pp. 262-276, 2015.

[15] N. D. Lagaros, S. George, and M. Papadrakakis, "A novel approach for the efficient simulation of highly skewed nonGaussian stochastic fields," in Proceedings of the European
Conference on Computational Mechanics, Lisbon, Portugal, June 2006.

[16] K. R. Gurley and A. Kareem, "A conditional simulation of non-normal velocity/pressure fields," Journal of Wind Engineering and Industrial Aerodynamics, vol. 77-78, pp. 39-51, 1998.

[17] R. J. Adler and J. E. Taylor, "Random fields and geometry," in Springer Monographs in MathematicsSpringer, New York, NY, US, 2007.

[18] A. Sancetta, "Nearest neighbor conditional estimation for Harris recurrent Markov chains," Journal of Multivariate Analysis, vol. 100, no. 10, pp. 2224-2236, 2009.

[19] Fu Zhou, Z. Ni, and Z. Xie, "Prediction of wind pressure field on pitched roof using POD," Chinese Quarterly of Mechanics, vol. 26, no. 2, pp. 248-255, 2005.

[20] D. Bosq, Linear Processes on Function Spaces: Theory and Applications, Springer-Verlag, New York, NY, US, 2000.

[21] V. Kargin and A. Onatski, "Curve forecasting by functional autoregression," Journal of Multivariate Analysis, vol. 99, no. 10 , pp. $2508-2526,2008$. 